\title{
Multi-class Posterior Atlas Formation via Unbiased Kullback-Leibler Template Estimation
}

\author{
Peter Lorenzen ${ }^{1}$, Brad Davis ${ }^{1}$, Guido Gerig ${ }^{1,2}$, Elizabeth Bullitt ${ }^{1,3,4}$, and \\ Sarang Joshi ${ }^{1,5}$ \\ Departments of ${ }^{1}$ Computer Science, ${ }^{2}$ Psychiatry, ${ }^{3}$ Surgery, ${ }^{4}$ Radiology, and \\ ${ }^{5}$ Radiation Oncology, \\ University of North Carolina, Chapel Hill, NC 27599, USA \\ \{lorenzen, davisb, gerig, joshi\}@cs.unc,edu, bullitt@med.unc.edu
}

\begin{abstract}
Many medical image analysis problems that involve multimodal images lend themselves to solutions that involve class posterior density function images. This paper presents a method for large deformation exemplar class posterior density template estimation. This method generates a representative anatomical template from an arbitrary number of topologically similar multi-modal image sets using large deformation minimum Kullback-Leibler divergence registration. The template that we generate is the class posterior that requires the least amount of deformation energy to be transformed into every class posterior density (each characterizing a multi-modal image set). This method is computationally practical; computation times grows linearly with the number of image sets. Template estimation results are presented for a set of five $3 \mathrm{D}$ class posterior images representing structures of the human brain.
\end{abstract}

\section{Introduction}

Computational anatomy, the study of anatomical variation, is an active area of research in medical image analysis. An important problem in computational anatomy is the construction of an exemplar template (or atlas) from a population of medical images. This template represents the anatomical variation present in the population [1,23]. Understanding anatomical variability requires robust high-dimensional image registration methods where the number of parameters used to describe the mappings between images is on the order of the number of voxels describing the space of the images.

Modern imaging techniques provide an array of imaging modalities which enable the acquisition of complementary information representing an underlying anatomy. Most image registration algorithms find a mapping between two scalar images. To utilize multi-modal images of a single anatomy, we define a multimodal image set, $\bar{I}$, as a collection of $m$ co-registered multi-modal images, $\bar{I}(x) \in$ $\mathbb{R}^{m}$. For example, $\bar{I}(x)$ might represent a CT image, a T1-weighted MR image, and a PET image of a single anatomy.

If, in traditional two-scalar image registration, the images are of different modalities, mutual information is typically used to register them. Highdimensional image registration, in the context of mutual information and other 
dissimilarity measure frameworks, has been studied extensively. A thorough investigation of these dissimilarity measures in high-dimensional image registration is presented in [4. A multi-modal free-form registration algorithm that matches voxel class labels, rather than image intensities, via minimizing Kullback-Leibler divergence is presented in [5]6]. This method finds correspondences between two multi-modal scalar images. A method that minimizes Kullback-Leibler divergence between expected and observed joint class histograms is presented in [7]. This technique, however, estimates class labels as a preprocessing step and is used only for rigid registration between scalar images. The method presented in this paper is more general in that registration is performed on sets of images, of arbitrary number and is not constrained by an initial class labeling. Although inter-subject high-dimensional image registration has received much attention 8 9,10 11, to our knowledge, little attention has been given to using multi-modal image sets of subjects to estimate registration transformations.

\subsection{Model-Based Multi-modal Image Set Registration}

Across image sets, the number of constituent images may vary, thus registration based on an intensity similarity measure is not possible in this setting. While mutual information can be extended to multiple random variables, its extension to registration involving three or more images is problematic in that it requires maintaining an impractical number of histogram bins [12. Given these difficulties, we move to a model-based approach where the registration is performed using underlying anatomical structures. We incorporate anatomical structures as a prior in a Bayesian framework as described in [13.

This framework is based on the assumption that human brain anatomy consists of finitely enumerable structures such as grey matter, white matter, and cerebrospinal fluid. These structures present with varying radiometric intensity values across disparate image modalities. Given a collection of multi-modal image sets representing the atlas population, we capture the underlying structures by estimating, for each image set, the class posterior densities associated with each of the structures. These class posterior densities are then used to produce the multi-class posterior atlas by estimating high-dimensional diffeomorphic registration maps relating the coordinate spaces of the densities. The Kullback-Leibler divergence is used as the distance function for the posterior densities to estimate the transformation. The use of the class posterior densities provides an image intensity independent approach to image registration.

\section{Bayesian Framework}

From a population of $N$ multi-modal image sets $\left\{\bar{I}_{i}\right\}_{i=1}^{N}$, for each class $c_{j}$ we first estimate the class posterior densities $p_{j}^{i}(x)=p\left(c_{j}(x) \mid \bar{I}_{i}\right)$ for each image set $i$ where $c_{j}(x)$ is the class associated with the voxel at position $x \in \Omega \subset$ $\mathbb{R}^{3}$. Again, this method is independent of the choice of the number of images comprising each image set. These class posterior densities are produced using 
the expectation maximization method described in [14,15]. Following [15,16], for each class $c_{j}$, the associated data likelihood, $p\left(\bar{I}_{i}(x) \mid c_{j}(x), \mu_{j}, \Sigma_{j}\right)$, is modeled as a normal distribution with mean, $\mu_{j}$, and covariance, $\Sigma_{j}$. The class posteriors are computed using a new atlas developed at UNC's department of psychiatry for two year old children. This atlas is based on fourteen subjects using the same concepts as used in the construction of the Montreal Neurological Institute (MNI) International Consortium for Brain Mapping (ICBM) atlas [17.

In this paper, we focus on the construction of an exemplar template from a population of anatomical class posterior densities. We use the method developed in [18] which provides an unbiased technique for atlas construction using large deformation diffeomorphic registration.

\section{Exemplar Templates}

We consider the problem of estimating a template class posterior $\hat{p}$ that is the best representative for a population of $N$ class posteriors, $\left\{p^{i}\right\}_{i=1}^{N}$, representing the $N$ individual image sets $\left\{\bar{I}_{i}\right\}_{i=1}^{N}$. The template $\hat{p}$ is not a member of the $\left\{p^{i}\right\}$. To this end, we consider the problem of constructing a mapping between $\hat{p}$ and each class posterior in the set $\left\{p^{i}\right\}$. That is, we estimate the mappings $h_{i}: \Omega \rightarrow \Omega_{i}$ where $\Omega \subset \mathbb{R}^{3}$ and $\Omega_{i} \subset \mathbb{R}^{3}$ are the coordinate systems of the class posteriors $\hat{p}$ and $p^{i}$ respectively. Again, $\Omega$ is independent of any of the population class posterior coordinate systems. This framework is depicted in

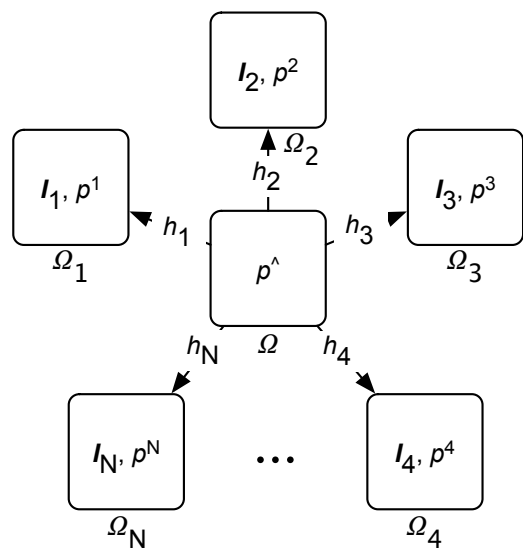

Fig. 1. Template Construction Framework Figure 1

Following the template construction framework developed in [18] we seek the representative template class posterior $\hat{p}$ that requires the minimum amount of energy to deform into every population class posterior $p^{i}$. More precisely, given a transformation group $\mathcal{S}$ with associated metric $D: \mathcal{S}^{2} \rightarrow \mathbb{R}$, along with a probability density dissimilarity measure $E(p, q)$, we wish to find the class posterior density $\hat{p}$ such that

$$
\left\{\hat{h}_{i}, \hat{p}\right\}=\underset{h_{i} \in \mathcal{S}, p}{\operatorname{argmin}} \sum_{i=1}^{N} E\left(p^{i} \circ h_{i}, p\right)+D\left(e, h_{i}\right)
$$

where $e$ is the identity transformation.

In this paper we focus on the infinite dimensional group of diffeomorphisms $\mathcal{H}$ as described in 18 . We apply the theory of large deformation diffeomor- 
phisms [9] to generate deformations $h$ that are solutions to the Lagrangian ODEs $\frac{d}{d t} h(x, t)=v(h(x, t), t)$.

We induce a metric on the space of diffeomorphisms by using a Sobolev norm (a norm involving derivatives of a function) via a partial differential operator $L$ on the velocity fields $v$. Let $h$ be a diffeomorphism isotopic to the identity transformation $e$. We define the distance $D(e, h)$ as

$$
D(e, h)=\min _{v} \int_{0}^{1} \int_{\Omega}\|L v(x, t)\|^{2} d x d t
$$

subject to

$$
h(x)=x+\int_{0}^{1} v(h(x, t), t) d t
$$

The distance between any two diffeomorphisms is defined by

$$
D\left(h_{1}, h_{2}\right)=D\left(e, h_{1}^{-1} \circ h_{2}\right) .
$$

The construction of $h$ and $h^{-1}$, as well as the properties of $D$, are described in 18 .

\section{Large Deformation Class Posterior Template Construction}

Having defined a metric on the space of diffeomorphisms, the minimum energy template estimation problem described in Equation 1 is formulated as

$$
\left\{\hat{h}_{i}, \hat{p}\right\}=\underset{h_{i}, p}{\operatorname{argmin}} \sum_{i=1}^{N} E\left(p^{i} \circ h_{i}, p\right)+\int_{0}^{1} \int_{\Omega}\left\|L v_{i}(x, t)\right\|^{2} d x t d
$$

subject to

$$
h_{i}(x)=x+\int_{0}^{1} v_{i}\left(h_{i}(x, t), t\right) d t .
$$

As a measure of dissimilarity between two probability density functions $p(x)$ and $q(x)$, at a spatial location $x$, we use the Kullback-Leibler divergence (relative entropy),

$$
D_{K L}(p(x), q(x))=\sum_{j=1}^{C} p_{j}(x) \log \frac{p_{j}(x)}{q_{j}(x)},
$$

where $C$ is the number of anatomical structure classes. From an information theoretic viewpoint [19, this dissimilarity can be interpreted as the inefficiency of assuming that an observation $q(x)$ is true when $p(x)$ is true. That is, we 
can use Kullback-Leibler divergence to measure how much the deformed class posteriors, $\left\{p^{i}\left(h_{i}(x)\right)\right\}_{i=1}^{N}$, deviate from the atlas $p(x)$.

Under the Kullback-Leibler divergence measure the template estimation problem becomes

$$
\hat{h}_{i}, \hat{p}=\underset{h_{i}, p}{\operatorname{argmin}} \sum_{i=1}^{N} \int_{\Omega} D_{K L}\left(p(x), p^{i}\left(h_{i}(x)\right)\right) d x+\int_{0}^{1} \int_{\Omega}\left\|L v_{i}(x, t)\right\|^{2} d x d t .
$$

This minimization problem can be simplified by noticing that for fixed transformations $h_{i}$, the $\hat{p}$ that minimizes Equation 2 is given by normalized geometric mean of the deformed class posteriors, $p^{i}(x)$,

$$
\hat{p}_{j}(x)=\frac{\left(\prod_{i=1}^{N} p_{j}^{i}\left(h_{i}(x)\right)\right)^{\frac{1}{N}}}{\sum_{k=1}^{C}\left(\prod_{i=1}^{N} p_{k}^{i}\left(h_{i}(x)\right)\right)^{\frac{1}{N}}} .
$$

Combining Equations 2 and 3 results in the following minimization problem

$$
\hat{h}_{i}=\underset{h_{i}}{\operatorname{argmin}} \sum_{i=1}^{N} \int_{\Omega} D_{K L}\left(\hat{p}(x), p^{i}\left(h_{i}(x)\right)\right) d x+\int_{0}^{1} \int_{\Omega}\left\|L v_{i}(x, t)\right\|^{2} d x d t .
$$

Note that the solution to this minimization problem is independent of the ordering of the $N$ image sets and increases linearly as image sets are added, thus, making the algorithm scalable.

\section{Implementation}

Following Christensen's algorithm for propagating templates described in [20], we approximate the solution to the minimization problem described in Equation 4 using an iteratively greedy method. At each iteration $n$, the updated transformation $h_{i}^{n+1}$, for each class posterior $p^{i}$, is computed using the update rule $h_{i}^{n+1}=h_{i}^{n}\left(x+\epsilon v_{i}^{n}(x)\right)$. The fields $h_{i}^{n}$ and $v_{i}^{n}$ are the current estimated transformations and the velocity for the $i$ th class posterior, and $\epsilon$ is the time step size. That is, each final transformation $h_{i}$ is built form the composition of $n$ transformations.

The velocity $v_{i}^{n}$ for each iteration $n$ is computed as follows. First, compute the updated template estimate (i.e. the normalized geometric mean)

$$
\hat{p}_{j}^{n}(x)=\frac{\left(\prod_{i=1}^{N} p_{j}^{i}\left(h_{i}^{n}(x)\right)\right)^{\frac{1}{N}}}{\sum_{k=1}^{C}\left(\prod_{i=1}^{N} p_{k}^{i}\left(h_{i}^{n}(x)\right)\right)^{\frac{1}{N}}}
$$

for each class component $j$. Next, following the second order approximation to 

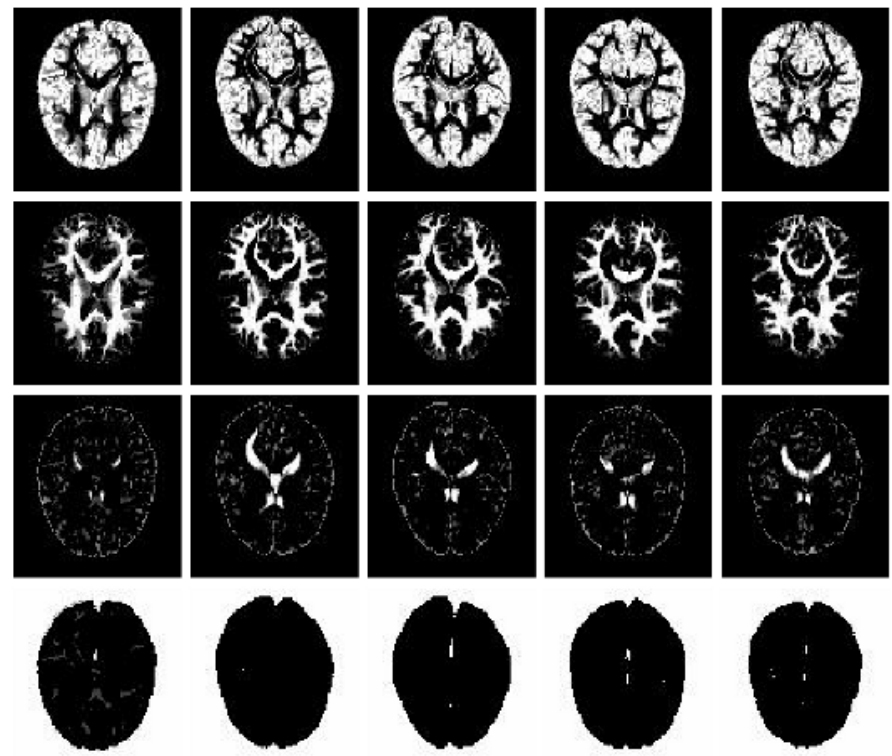

Fig. 2. Five class posteriors each with three classes and a background class. These images clearly show the large inter-subject variability, especially in the ventricular system.

Kullback-Leibler divergence described in [13] define the body force functions

$$
F_{i}^{n}(x)=\sum_{k=1}^{C}\left[\frac{p_{k}^{i}\left(h_{i}(x)\right)}{\hat{p}_{k}(x)}-2\right] \nabla p_{k}^{i} \mid \begin{aligned}
& T \\
& h_{i}(x)
\end{aligned} .
$$

This is the variation of the class posterior dissimilarity term in Equation 4 with respect to the transformation $h_{i}$. The velocity field $v_{i}^{n}$ is computed at each iteration by applying the inverse of the differential operator $L$ to the body force function, that is, $v_{i}^{n}(x)=L^{-1} F_{i}^{n}(x)$, where $L=\alpha \nabla^{2}+\beta \nabla \cdot \nabla+\gamma$ is the Navier-Stokes operator. This computation is performed in the Fourier domain [21].

\section{Results}

To evaluate the performance of this method we applied the algorithm to a set of five class posterior densities that were derived from a population of T1-weighted, T2-weighted, and proton density 3D MR images of brains of health two year old children using an expectation maximization segmentation method [1516]. As a preprocessing step, these images were aligned using affine registration. An axial slice from each derived class posterior density is shown in Figure 2. There is noticeable variation between these anatomies, especially in the ventricular region. 

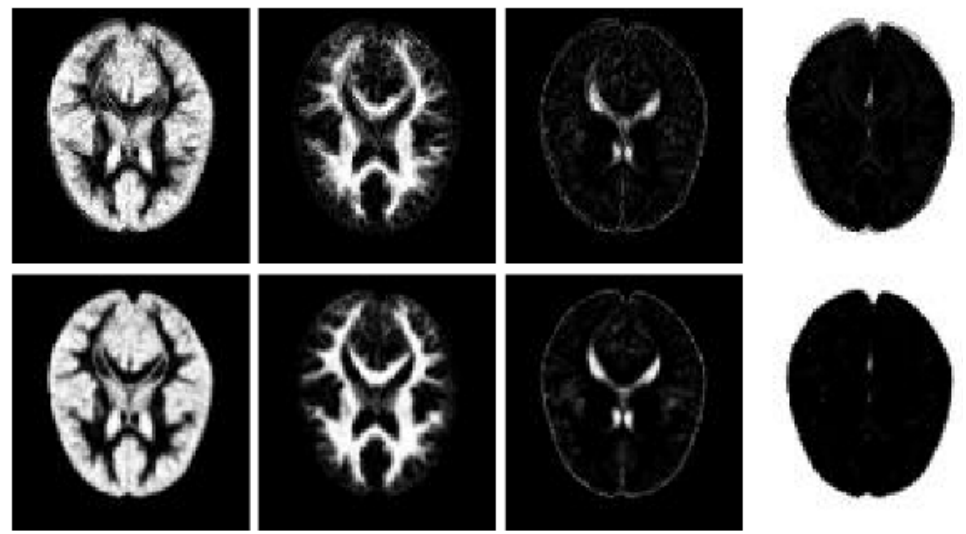

Fig. 3. Template Construction. The top row shows the normalized geometric mean class posterior density following an affine registration of all five subjects. The bottom row represents the estimated template after the final iteration of the algorithm.

Figure 3 shows the normalized geometric mean of the five class posterior densities following the affine registration and the final estimate of the template. The normalized geometric mean is blurry since it is an "average" of the varying individual neuroanatomies. Ghosting is evident around the lateral ventricles and near the boundary of the brain. In the final estimate of the template these variations have been accommodated by the high-dimensional registration.

Acknowledgments. The authors would like to thank Matthieu Jomier for producing the class posterior densities and Dr. Mark Foskey for insightful discussions. This work was supported by NIBIB-NIH grant R01 EB000219, DOD Prostate Cancer Research Program DAMD17-03-1-0134, NIMH grant MH064580 Longitudinal MRI Study of Brain Development in Fragile X, and the NDRC.

\section{References}

1. Miller, M., Banerjee, A., Christensen, G., Joshi, S., Khaneja, N., Grenander, U., Matejic, L.: Statistical methods in computational anatomy. Statistical Methods in Medical Research 6 (1997) 267-299

2. Grenander, U., Miller, M.: Computational anatomy: an emerging discipline. Quarterly of Applied Mathematics 56 (1998) 617-694

3. Thompson, P., Woods, R., Mega, M., Toga, A.: Mathmatical/computational challenges in creating deformable and probabilistic atlases of the human brain. Human Brain Mapping 9 (2000) 81-92

4. Hermosillo, G.: Variational Methods for Multimodal Image Matching. PhD thesis, Universite de Nice - Sophia Antipolis (2002)

5. D'Agostino, E., Maes, F., Vandermeulen, D., Suetens, P.: A viscous fluid model for multimodal non-rigid image registration using mutual information. Medical Image Analysis (MedIA) 7 (2003) 565-575 
6. D'Agostino, E., Maes, F., Vandermeulen, D., Suetens, P.: An information theoretic approach for non-rigid image registration using voxel class probabilities. In: International Workshop on Biomedical Image Registration (WBIR). Volume 2717 of Lecture Notes in Computer Science (LNCS)., Springer-Verlag (2003) 122-131

7. Chan, H.M., Chung, A.C.S., Yu, S.C.H., Norbash, A., Wells W.M. III, Multi-modal image registration by minimizing Kullback-Leibler distance between expected and observed joint class histograms. Proceedings of the IEEE Computer Vision and Pattern Recognition (CVPR) 2 (2003) 570-576

8. Rueckert, D., Hayes, C., Studholme, C., Summers, P., Leach, M., Hawkes, D.J.: Non-rigid registration of breast mr images using mutual information. In: Proceedings of Medical Image Computing and Computer-Assisted Intervention (MICCAI). Lecture Notes in Computer Science (LNCS), Springer-Verlag (1998) 1144-1152

9. Miller, M.I., Joshi, S.C.: Large deformation fluid diffeomorphism for landmark and image matching. In: Brain Warping. Academic Press, San Diego (1999) 115-131

10. Gaens, T., Maes, F., Vandermeulen, D., Suetens, P.: Non-rigid multimodal image registration using mutual information. In: Proceedings of Medical Image Computing and Computer-Assisted Intervention (MICCAI). Lecture Notes in Computer Science (LNCS), Springer-Verlag (1998) 1099-1106

11. Studholme, C., Hill, D.L.G., Hawkes, D.J.: An overlap invariant entropy measure of 3d medical image alignment. Pattern Recognition (1998) 71-86

12. Bhatia, K.K., Hajnal, J.V., Puri, B.K., Edwards, A.D., Rueckert, D.: Consistent groupwise non-rigid registration for atlas construction. In: Proceedings of IEEE International Symposium on Biomedical Imaging (ISBI), IEEE (2004) 908-911

13. Lorenzen, P., Joshi, S.: High-dimensional multi-modal image registration. In: International Workshop on Biomedical Image Registration (WBIR). Volume 2717 of Lecture Notes in Computer Science (LNCS)., Springer-Verlag (2003) 234-243

14. Moon, N., van Leemput, K., Gerig, G.: Automatic brain and tumor segmentation. In: Medical Image Computing and Computer-Assisted Intervention (MICCAI). Volume 2489 of Lecture Notes in Computer Science (LNCS)., Tokyo, SpringerVerlag (2002) 372-379

15. van Leemput, K., Maes, F., Vandermeulen, D., Suetens, P.: Automated modelbased tissue classification of $\mathrm{mr}$ images of the brain. IEEE Transactions on Medical Imaging (TMI) 18 (1999) 897-908

16. van Leemput, K., Maes, F., Vandermeulen, D., Suetens, P.: Automated modelbased bias field correctionof mr images of the brain. IEEE Transactions on Medical Imaging (TMI) 18 (1999) 885-896

17. Cocosco, C., Kollokian, V., Kwan, R.S., Evans, A.: Brainweb: Online interface to a $3 \mathrm{~d}$ mri simulated brain database. In: NeuroImage (Proceedings of 3-rd International Conference on Functional Mapping of the Human Brain). Volume 5., Copenhagen (1997) S425

18. Davis, B., Lorenzen, P., Joshi, S.: Large deformation minimum mean squared error template estimation for computation anatomy. In: Proceedings of IEEE International Symposium on Biomedical Imaging (ISBI), IEEE (2004) 173-176

19. Cover, T., Thomas, J.: Elements of Information Theory. John Wiley \& Sons, Inc., New York (1991)

20. Christensen, G., Rabbit, R., Miller, M.: Deformable templates using large deformation kinematics. Transactions on Image Processing 5(10) (1996) 1435-1447

21. Joshi, S., Lorenzen, P., Gerig, G., Bullitt, E.: Structural and radiometric asymmetry in brain images. Medical Image Analysis (MedIA) 7 (2003) 155-170 